\title{
BMJ Open Botulinum toxin treatment of spasticity targeted to muscle endplates: an international, randomised, evaluator- blinded study comparing two different botulinum toxin injection strategies for the treatment of upper limb spasticity
}

To cite: Rekand T, BieringSörensen B, He J, et al. Botulinum toxin treatment of spasticity targeted to muscle endplates: an international, randomised, evaluator-blinded study comparing two different botulinum toxin injection strategies for the treatment of upper limb spasticity. BMJ Open 2019;9:e024340. doi:10.1136/ bmjopen-2018-024340

- Prepublication history and additional material for this paper are available online. To view these files, please visit the journal online (http://dx.doi. org/10.1136/bmjopen-2018024340).

Received 22 May 2018 Revised 17 December 2018 Accepted 25 February 2019

Check for updates

(c) Author(s) (or their employer(s)) 2019. Re-use permitted under CC BY-NC. No commercial re-use. See rights and permissions. Published by BMJ.

For numbered affiliations see end of article.

Correspondence to

Dr Tiina Rekand;

tiina.rekand@helse-bergen.no

Tiina Rekand, ${ }^{1}$ Bo Biering-Sörensen, ${ }^{2}$ Jun $\mathrm{He},{ }^{3}$ Ole Jakob Vilholm, ${ }^{4}$ Peter Brøgger Christensen, ${ }^{5}$ Trandur Ulfarsson, ${ }^{6}$ Roger Belusa, ${ }^{7}$ Torbjörn Ström, ${ }^{7}$ Peter Myrenfors, ${ }^{7}$ Pascal Maisonobe, ${ }^{7,8}$ Torben Dalager $^{9}$

\section{ABSTRACT}

Objectives The therapeutic effects of botulinum neurotoxin (BoNT) are well documented in upper limb spasticity. However, several factors may influence treatment efficacy, including targeting of neuromuscular junctions (NMJs). We examined whether NMJ-targeted BoNT injections were non-inferior, in terms of efficacy, to current injection practices.

Design Open-label prospective evaluator-blinded study. Setting Conducted across 20 medical centres in Denmark, Finland, Norway and Sweden (24 September 2012 to 11 March 2015).

Participants Aged $>18$ years with upper limb spasticity (Modified Ashworth Scale [MAS] score of 2 or 3 ) following stroke or traumatic brain injury, had received $\geq 2$ consecutive BoNT-A treatment cycles (the latest of which was abobotulinumtoxinA [aboBoNT-A]) and needed BoNT-A retreatment (same modality as previous cycle). Patients requiring aboBoNT-A doses $>800$ units were excluded. In total, 88 patients were randomised (intentionto-treat [ITT] population), most were male $(n=58 / 88$, $65.9 \%$ ) and $54 / 88(61.4 \%)$ completed the study (per protocol [PP] population).

Interventions Randomisation (1:1) to receive a single dose of aboBoNT-A ( $\leq 800 \mathrm{U})$ according to either current clinical practice $(300 \mathrm{U} / \mathrm{mL})$ or as an NMJ-targeted injection $(100 \mathrm{U} / \mathrm{mL})$.

Primary outcome measure Proportion of patients with a $\geq 1$ level reduction from baseline in MAS score at week 4 post-injection (responders).

Results In the ITT population, the proportion of responders at elbow flexors was $72.7 \%$ in the current practice group and $56.8 \%$ in the NMJ-targeted group (adjusted difference -0.1673 [95\% Cls: -0.3630 to $0.0284] ; p=0.0986$ ). Similar results were observed in the PP population ( $69.0 \%$ vs $68.0 \%$, respectively, adjusted difference 0.0707 [ -0.1948 to 0.3362$] ; p=0.6052$ ). Conclusions Owing to the limited number of participants, non-inferiority of NMJ-targeted injections could not be determined. However, there was no statistical difference
Strengths and limitations of this study

This study contributes to the ongoing discussion around the significance of targeted injections in botulinum neurotoxin treatment.

The study failed to reach its recruitment target, preventing the demonstration of statistically significant non-inferiority.

- Due to the heterogenic population, a cross-over design might have given a stronger power and been able to demonstrate non-inferiority.

- Even though recruitment was terminated early, this study is one of the larger evaluator-blinded prospective randomised studies performed investigating the neuromuscular junction targeting treatment technique.

- Modified Ashworth Scale was used as the primary outcome measure in this study, it is possible that minor changes were not captured by this score, suggesting that future studies should consider using additional methods of evaluations, such as eletromyography.

between groups. Larger studies are needed confirm whether the two techniques offer comparable efficacy. Trial registration number NCT01682148.

\section{INTRODUCTION}

Spasticity is a sensorimotor control disorder, resulting from an upper motor neuron lesion, presenting as intermittent or sustained involuntary activation of muscles. ${ }^{1}$ Patients with spasticity may suffer from decreased active movement, increased disability and impaired function. ${ }^{2}$ Intramuscular injections with botulinum neurotoxin (BoNT) is a documented efficacious and well-tolerated treatment 
option for these patients, which has an established position in clinical practice. ${ }^{3}{ }^{4}$ Studies with abobotulinumtoxinA (aboBoNT-A, Dysport) have demonstrated a beneficial effect on spasticity in terms of decreased resistance to passive movement, improvements in pain and goal attainment as well as in active function. ${ }^{5-10}$ The therapeutic effects of BoNT have been extensively studied in upper limb spasticity, which has been summarised in a number of recent reviews. ${ }^{11-13}$ Several factors can influence treatment efficacy, such as injection technique, targeting of neuromuscular junctions (NMJs), concentration of BoNT and volume of injected solution, as well as stimulation of BoNT uptake in target cells. ${ }^{14} 15$ Mapping of NMJ zones in the upper limb flexor muscles has enabled targeting of these zones to potentially increase the efficacy of BoNT injection in spasticity treatment. ${ }^{16-18}$ Indeed, results from studies in both animals and humans indicate that targeting NMJ zones can increase the effectiveness of BoNT injections. ${ }^{19-21}$ Gracies et $a l^{20}$ suggested that high volume or endplate targeted BoNT injections result in superior neuromuscular blockade, contraction and spasticity reduction than low volume, non-targeted injections. ${ }^{20}$ This study explores the hypothesis that the effect of a single high volume aboBoNT-A injection of low concentration per muscle, if targeted to the area of the NMJ zones, is equal to the effect of several injections using standard injection practices. An international randomised, multicenter, evaluator-blinded study was thus performed to evaluate non-inferiority between the two different injection strategies.

\section{MATERIALS AND METHODS}

\section{Study design}

The NMJ study was an international, multicenter, prospective, randomised, evaluator-blinded, parallel-group study, comparing two different injection techniques. Patients were injected with botulinum toxin (aboBoNT-A, Dysport) for the treatment of upper limb spasticity. Patients were randomised to 1 of 2 groups: (1) the current practice group receiving injections according to current clinical practice (without regard to NMJ) and (2) the NMJ-targeted group receiving injections according to a predefined NMJ targeting technique with lower concentration of the toxin (figure 1).

\section{Patient and public involvement}

In this study, the patient-centric research question was developed with the aim of limiting the injection points and thus limiting burden of injections. The patient's experience of the different injection techniques was recorded by measuring injection pain using a Visual Analogue Scale (VAS). Evaluation of outcome measures was via the patient-centric Goal Attainment Scaling (GAS) score where the patient and treating physician agree on a goal that is meaningful for the patient, and evaluate the treatment by goal attainment.

Patients were asked whether they would like to participate in the study and decided if they wanted to participate or not after provision of informed consent. When completing the informed consent form, patients could indicate whether they wished to be informed of the study results or not. Patients wishing to know the results were informed by their investigator.

\section{Patient recruitment}

Medical centres in Finland, Denmark, Sweden and Norway $(n=39)$ were included to recruit patients for participation in the study. Approval from the local ethical review board was granted in each separate country. The study conformed to the principles of the Helsinki Declaration and all patients signed an informed consent form ahead of participation.

Inclusion criteria included age $>18$ years, provision of written informed consent and suffering from upper limb spasticity position pattern type 1,3 or 4 following stroke or traumatic brain injury. ${ }^{22}$ The position patterns were based on characteristic arm positions and postures recognised in upper limb spasticity. ${ }^{23}$ Spasticity was evaluated according to the Modified Ashworth Scale (MAS),

Screening, randomisation
and treatment
Visit 1 (day 1)

Treatment
follow-up
Visit 2 (week 4)

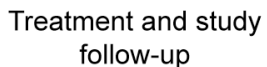

Visit 3 (week 12)

Figure 1 Study design. *If no routine BoNT-A treatment was given at visit 3, max 24 weeks poststudy injection. NMJ, neuromuscular junction. 
with a baseline score of 2 or 3 points at inclusion. Patients had received at least two consecutive previous treatment cycles with BoNT-A, of which at least the latest had to have been with aboBoNT-A with observed good efficacy according to investigator judgement. Furthermore, the investigator should have judged a need for a repeat of the same treatment modality as the previous cycle and the last BoNT-A treatment should have been dated $>12$ weeks before study entry. Subjects were randomised (1:1) to 1 of 2 treatment groups. The patients were stratified according to three different arm spasticity position patterns (1,3 and 4) and country of residence, aiming at a balanced ratio regarding these parameters.

Exclusion criteria included a need for doses of $>800 \mathrm{U}$ aboBoNT-A in the upper limb, a likelihood of changing other spasticity treatment (such as physiotherapy) during the study period, other neurological disorders or use of agents known to interfere with neuromuscular transmission. For full inclusion/exclusion criteria, see ClinicalTrials.gov).

Recruitment dates were first subject in 24 September 2012, last subject in 16 December 2014 and last subject in 11 March 2015.

\section{Randomisation procedure}

A randomisation list was prepared by a statistician independent from the study, and was provided to the electronic version of the Case Report Form (eCRF) provider who incorporated it into the eCRF. The list was stratified for three arm spasticity position patterns (1,3 and 4) and for participating countries, and generated with a balanced 1:1 treatment ratio. Once eligibility was confirmed, the investigator entered eligibility data into the eCRF. If the patient was eligible, based on the predefined randomisation list, the eCRF system would automatically assign the first not-used randomisation number corresponding to the patient's spasticity pattern and country, to get the applicable treatment administration group allocation. The study drug was provided in $3 \mathrm{~mL}$ vials containing 300 $\mathrm{U}$ of aboBoNT-A and diluted by the investigator to the assigned concentration, 100 or $300 \mathrm{U} / \mathrm{mL}$ according to the assigned treatment group.

\section{Blinding}

This was an open study with respect to study drug. However, the treatment group assigned to a randomisation number was to be blinded prior to allocation. To optimise protocol blindness at each site, all study injections were to be performed by one of the investigators (unblinded) and all efficacy assessments were to be performed by another qualified study personnel, blinded to the injection technique. The eCRF provided two user profiles, one which was able to see the randomisation information (treatment arm) and another one for the evaluators, for which the treatment arm was hidden.

\begin{tabular}{|c|c|c|}
\hline Injection site & $\begin{array}{l}\text { Current } \\
\text { practice }\end{array}$ & NMJ-targeted \\
\hline m. biceps brachii* & $2-4$ & 1 \\
\hline m. brachialis* & $2-4$ & 1 \\
\hline m. brachioradialis* & $1-2$ & 1 \\
\hline $\begin{array}{l}\text { m. flexor carpi } \\
\text { radialis/m. flexor } \\
\text { carpi ulnaris* }^{\star}\end{array}$ & $2-3$ & 1 \\
\hline Volume of injection & $\begin{array}{l}0.1-0.7 \mathrm{~mL} \text { per } \\
\text { muscle }\end{array}$ & $\begin{array}{l}0.4-2.0 \mathrm{~mL} \text { per } \\
\text { muscle }\end{array}$ \\
\hline $\begin{array}{l}\text { Concentration of } \\
\text { aboBoNT-A }\end{array}$ & $300 \mathrm{U} / \mathrm{mL}$ & $100 \mathrm{U} / \mathrm{mL}$ \\
\hline
\end{tabular}

*Number of injection points.

aboBoNT-A, abobotulinumtoxinA.

\section{Treatments}

Optimal dose of aboBoNT-A (Dysport) for each subject was determined by the investigator at the first visit to optimise treatment efficacy prior to inclusion (see the Patient recruitment section). Maximum dose injected in the spastic arm was $800 \mathrm{U}$. Patients in the current practice group received injections according to current clinical practice (toxin concentration of $300 \mathrm{U} / \mathrm{mL}$ ). The NMJ-targeted group were injected using an NMJ targeted technique (low toxin concentration of $100 \mathrm{U} / \mathrm{mL}$ ). All injections were targeted to the same muscles of the arm and performed with support of electromyography (EMG) guiding and/or ultrasound (US). The study treatment was performed using the same individual dose as determined at the pre-study treatment visit.

The two different injection patterns are outlined in table 1. Each patient visited the clinic on at least three occasions. The first visit was devoted to screening, randomisation and treatment with aboBoNT-A. The second visit was scheduled 4 weeks after the first injection. Lastly, the third visit was a follow-up at 12 weeks after the first injection. Additional treatment with aboBoNT-A was given if necessary.

For the group treated with NMJ-targeted injections, the localisation of the single injection was based on previously published descriptions of the motor endplate bands within human arm muscles in relation to external landmarks. $^{2425}$

Assessment of arm spasticity was based on flexion of the elbow joint as well as muscle activity specific to position patterns 1, 3 and 4, as previously described by Hefter et $a .^{23}$

\section{Assessments and outcome measures}

The aim of the study was to assess non-inferiority of a treatment strategy using fewer injections of low concentration BoNT, as compared with current clinical practice. The primary endpoint was percentage of patients with at least a 1 level decrease in MAS (scored between 0 and 4). Baseline MAS score in the elbow flexors was 
compared with MAS score measured 4 weeks post injection. A $\geq 1$ level decrease in MAS from baseline was considered clinically meaningful. ${ }^{26}$ Secondary endpoints included change in MAS score in the elbow flexors from baseline to week 12 , intensity of spasticity related pain as measured by a VAS (scored 0-100) at baseline, week 4 and week 12, pain from injection measured by a VAS at first visit, score on the GAS (scored from -2 to 2 ) at week 4 or week 12, subject global evaluation of treatment effect at the study end and investigator preference of injection technique.

\section{Sample size and statistics}

Statistical methods: both the primary endpoint (MAS responders at week 4) and the non-inferiority design were taken into account for the sample size calculation. A two-sided 95\% CI for the difference in clinical success rates was calculated using a generalised linear model with treatment, spasticity position patterns, country and baseline MAS score as factors. Assuming a responder rate (ळ) of $63 \%$ in the reference group, a sample size of 122 patients per group would provide $80 \%$ power to detect a clinically relevant delta $(\Delta)$ of $17 \%$ at an alpha $(\alpha)$ level of $2.5 \%$ (one-sided). Assuming a dropout rate of $10 \%, 136$ patients per group were required to be randomised (ie, 272 patients in total).

The statistical analyses were performed in accordance with ICH E9 guideline and were based on the pooled data from the individual study sites, unless otherwise stated. All statistical analyses were performed by PCG using SAS V.9.4.

\section{RESULTS}

\section{Patient population}

The study was terminated early owing to slow recruitment, meaning the final population size in the two treatment arms did not reach the preplanned population size of 272 subjects. The main reasons for this were recruitment difficulties and protocol violations. In all, 20 centres included a total of 88 patients who were subsequently randomised in the study (intention_to-treat [ITT] population). However, only 54 of these completed the study without protocol violation (per protocol [PP] population) (online supplementary figure 1). Violation of protocol was recognised for 34 patients. Of these, 23 were not injected as PP and 11 had other violations, for example, 5 patients had missing MAS evaluations. Baseline characteristics for the ITT and PP populations for both aboBoNT-A treatment regimens (current practice vs NMJ-targeted) are shown in table 2.

\section{Primary endpoint}

The proportion of MAS responders (defined as $\geq 1$ level decrease in MAS from baseline) in the ITT population for elbow flexors at week 4 post-injection was $72.7 \%$ in the group treated as current practice and $56.8 \%$ in the group treated with NMJ-targeted injections (figure 2). The adjusted difference in clinical success rates between the two treatment regimens (group treated with NMJ-targeted injections minus group treated as current practice) was $-0.1673(95 \%$ CI $[-0.3630$ to 0.0284$], \mathrm{p}=0.0986)$ in favour of the group treated as current practice. Mean (SD) changes in MAS score for elbow flexors at week 4 compared with baseline are presented in figure 3 (ITT population).

Table 2 Baseline characteristics

\begin{tabular}{|c|c|c|c|c|}
\hline \multirow[b]{2}{*}{ Variable } & \multicolumn{2}{|l|}{ ITT population } & \multicolumn{2}{|l|}{ PP population } \\
\hline & Current practice & NMJ-targeted & Current practice & NMJ-targeted \\
\hline Number of subjects & 44 & 44 & 29 & 25 \\
\hline Male, n (\%) & $28(-63.60)$ & $30(-68.20)$ & $18(-62.10)$ & $17(-68.00)$ \\
\hline \multirow{2}{*}{$\begin{array}{l}\text { Spasticity patterns \% } \\
(\mathrm{I}, \mathrm{III}, \mathrm{IV})\end{array}$} & 9.1 & 11.4 & 6.9 & 12.0 \\
\hline & 36.4 & 29.5 & 41.4 & 40 \\
\hline $\begin{array}{l}\text { Years since stroke/injury } \\
\text { (median, range) }\end{array}$ & $4(1-21)$ & $4(1-24)$ & $3(1-16)$ & $5.5(1-24)$ \\
\hline $\begin{array}{l}\text { Total aboBoNT-A units } \\
\text { (mean } \pm \text { SD) }\end{array}$ & $566.4 \pm 169.8$ & $588 \pm 200.3$ & $537.8 \pm 130.2$ & $579.4 \pm 180.5$ \\
\hline Ultrasound & 0 & $1(2.4 \%)$ & 0 & 0 \\
\hline Missing & 0 & 2 & 1 & 0 \\
\hline
\end{tabular}

aboBoNT-A, abobotulinumtoxinA; ITT, intention-to-treat; NMJ, neuromuscular junction; PP, per protocol. 


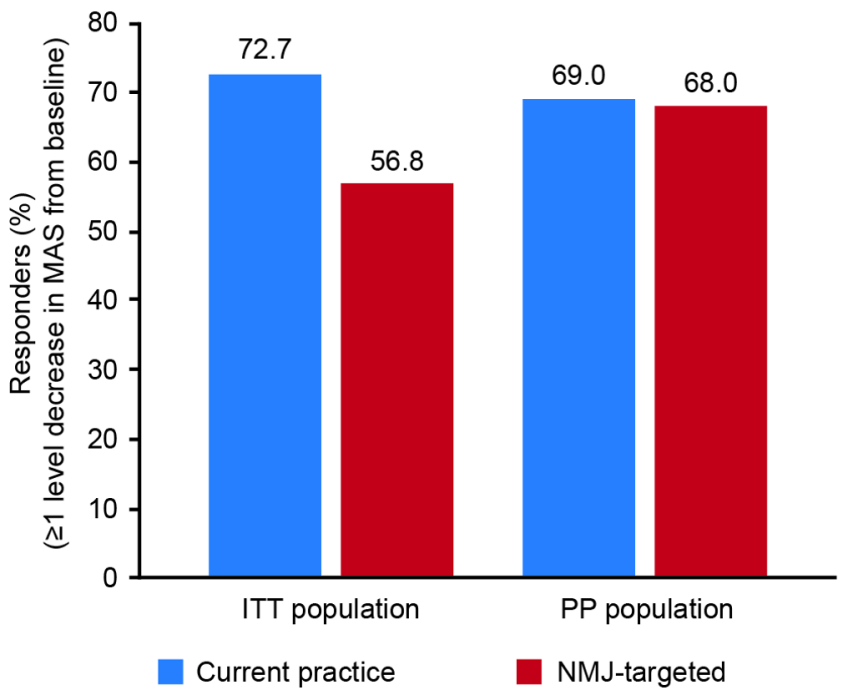

Figure 2 Proportion (\%) of responders at week 4 ( $\geq 1$ level decrease in MAS from baseline). Adjusted difference of ratios for each population (95\% Cl): ITT: -0.1673 $(-0.3630$ to 0.0284$) p=0.0986$; PP: 0.0707 ( -0.1948 to 0.3362$)$ $\mathrm{p}=0.6052$. Non-inferiority was not demonstrated. ITT, intention-to-treat; MAS, Modified Ashworth Scale; NMJ, neuromuscular junction; PP, per protocol.

Proportion of responders (defined as $\geq 1$ level decrease in MAS from baseline) in the PP population for elbow flexors at week 4 was $69.0 \%$ in the group treated as current practice and $68.0 \%$ in the group treated with NMJ-targeted injections (95\% CI of the adjusted difference $[-0.1948$ to 0.3362$], \mathrm{p}=0.6052)$, as outlined in figure 2. There were no significant differences observed between groups. Non-inferiority was not shown in either population. Mean (SD) changes in MAS score for elbow flexors at week 4 compared with baseline are presented in figure 3 .

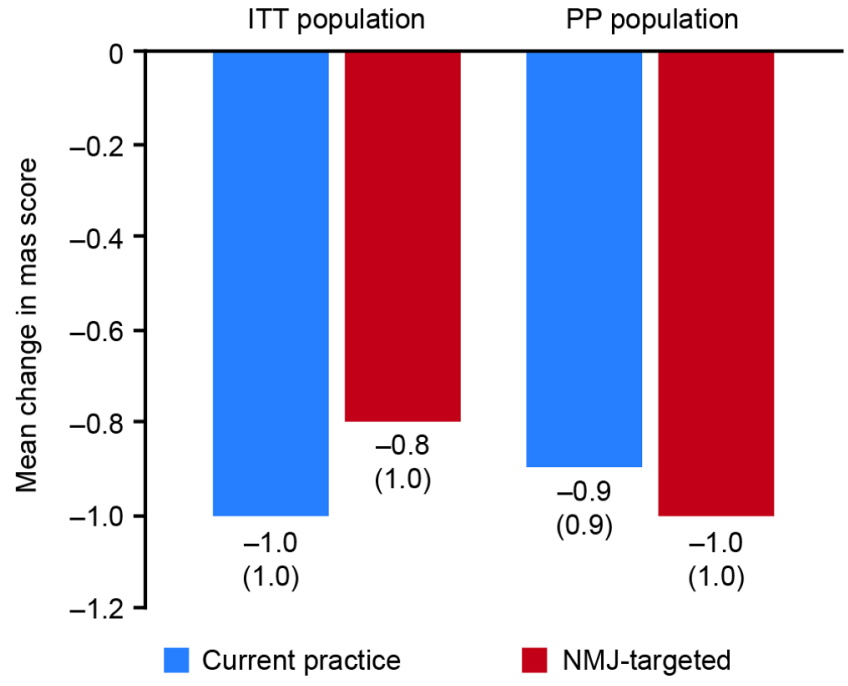

Figure 3 Mean (SD) change in MAS in elbow flexors at week 4compared with baseline. ITT, intention-to-treat; MAS, Modified Ashworth Scale; NMJ, neuromuscular junction; PP, per protocol.

\section{Secondary endpoints}

Secondary endpoints evaluated were MAS responders at 12 weeks, improvement in GAS score as well as spasticity pain between baseline and 4 weeks and injection related pain at first visit. All secondary endpoints were measured on the ITT population. No secondary endpoint showed any significant difference between the two groups. Results from the secondary endpoints are summarised in table 3.

\section{Safety}

The adverse events reported were consistent with the known safety profile of aboBoNT-A and no new adverse effects were observed. Treatment emergent adverse events (TEAEs) were experienced in 11 subjects in the group treated as current practice $(25.0 \%$; 16 events were reported) and 15 in the group treated with NMJ-targeted injections $(34.1 \%$; 24 events were reported). For all subjects $(n=88)$, the TEAEs reported by more than one subject were urinary tract infection (reported by four subjects, $4.5 \%$ ), headache and epilepsy (both reported by three subjects, $3.4 \%$ ) and pain in extremity, epididymitis and fatigue (all three reported by two subjects, 2.3\%). Regarding investigator assessed TEAEs related to treatment, three events were reported in two subjects in the group treated as current practice $(4.5 \%)$ and one event was reported in the group treated with NMJ-targeted injections (2.3\%). In the current practice group, these concerned one patient with injection site hypersensitivity, which was assessed as mild and study treatment related, and one patient with dysphagia and fatigue, both of which were assessed as mild and study treatment related. In the NMJ-targeted group, one patient experienced fatigue, assessed as moderate and related to the study treatment. Overall, the majority of events reported were mild to moderate in intensity. Additionally, in the NMJ-targeted group, three subjects experienced severe TEAEs which were assessed as non-related to aboBoNT-A treatment by the investigator. Two subjects in each group experienced serious adverse events judged by the investigator as non-related to the treatment. There were no reported TEAEs resulting in withdrawal or death.

\section{DISCUSSION}

In the current study, both injection techniques using aboBoNT-A resulted in a high proportion of responders, while no statistically significant difference between the injection strategies was observed. However, the number of subjects included in the study was a limiting factor and may explain the failure to demonstrate non-inferiority statistically. Previously, the injection strategy used for BoNT treatment has been identified as important for optimising treatment outcomes. ${ }^{142728}$ Indeed, NMJ-targeted strategies have the advantage of requiring fewer injection points, potentially minimising pain associated with administration and are expected to be preferable for both patients and physicians. This study contributes to the discussion about the significance of targeted injections. 
Table 3 Evaluation of secondary endpoints in the ITT population.

\begin{tabular}{|c|c|c|}
\hline & Current practice & NMJ-targeted \\
\hline & $n=44$ & $n=44$ \\
\hline \multicolumn{3}{|l|}{ MAS response at 12 weeks } \\
\hline n (\%) & $19(48.7 \%)^{\star}$ & $13(33.3 \%) \dagger$ \\
\hline Adj difference & & $-0.1324 ; 95 \% \mathrm{Cl}(-0.3531$ to 0.0884$)$ \\
\hline$P$ value & & 0.24 \\
\hline \multicolumn{3}{|l|}{ GAS score improvement } \\
\hline $\mathrm{n}(\%)$ & $25(61.0 \%) \ddagger$ & $21(63.6 \%) \S$ \\
\hline$P$ value & & 0.5747 \\
\hline \multicolumn{3}{|c|}{ Spasticity related pain, VAS $($ mean $\pm S D)$} \\
\hline Baseline & $14.32 \pm 22.64$ & $21.66 \pm 28.97$ \\
\hline Week 4 & $10.30 \pm 19.99 \rrbracket$ & $14.05 \pm 27.50^{\star \star}$ \\
\hline Mean change & $-4.35 \pm 12.29$ & $-5.8 \pm 23.07$ \\
\hline Decrease of mean (\%) & 30.4 & 26.8 \\
\hline \multicolumn{3}{|c|}{ Injection-related pain VAS (at first visit) } \\
\hline Mean \pm SD & $30.68 \pm 27.33^{\star \star}$ & $25.67 \pm 25.37 \dagger \dagger$ \\
\hline Difference & & -5.01 \\
\hline$P$ value & & 0.406 \\
\hline
\end{tabular}

*5 patients missing 12-week MAS evaluation.

†5 patients missing 12-week MAS evaluation.

$\ddagger 3$ patients missing GAS evaluation.

$\S 11$ patients missing GAS evaluation.

I1 patient missing VAS evaluation.

${ }^{\star *} 7$ patients missing VAS evaluation.

††8 patients missing VAS evaluation.

GAS, Goal Attainment Scaling; MAS, Modified Ashworth Scale; NMJ, neuromuscular junction; VAS, Visual Analogue Scale.

Assessment of the ability for passive movements, as measured by MAS, did not show any significant differences between the groups, at 4-week or at 12-week follow-up. Generally, injection techniques based on a lower number of injection points, as in the NMJ strategy, are expected to be preferable for both patients and physicians.

Gracies $e t a l^{20}$ suggested that high volume or endplate targeted BoNT injections results in superior neuromuscular blockade, contraction and spasticity reduction than low volume, non-targeted injections. ${ }^{20}$ In the Gracies study, the effect was demonstrated using the Tardieu scale in contrast to the MAS used in the current study. Hence, the results are not directly comparable. The lack of statistically significant differences between the strategies in our study might, however, be explained by the different outcome measures used. It might not be possible to capture a statistical difference with the MAS scale in this limited study group, due to lack of power. The GAS, which is a more sensitive scale than MAS to investigate functionally important changes after injection,${ }^{29}$ generated similar results between the strategies used in our study.

The experienced pain related to spasticity or injection was mild or moderate. A reduction in pain intensity after 4 weeks was observed in both treatment strategies. Pain associated with injections of BoNT could hypothetically influence optimal treatment results and goal attainment by affecting compliance. An inverse relationship between injection site pain and compliance to BoNT treatment has, for instance, been reported for hyperhidrosis. ${ }^{30}$

The study did not disclose any previously unknown adverse events. Furthermore, there was no difference in the observed frequency of adverse events between the two injection strategies, as assessed in the ITT population.

The major limitation of this study was the low number of included subjects, as compared with the number needed according to the calculation of statistical power conducted before the study. Most likely, this prevented statistical demonstration of non-inferiority. The main reasons underlying the slow recruitment were difficulties in finding patients who were receiving continuous treatment who also had a MAS score of 2 or higher in the elbow flexors. Also, patients treated successfully with BoNT-A did not fluctuate to the extent that they increased a full level on the MAS between injections. A further limitation was the fact that although patient priorities and preferences were considered when developing the study, patients were not consulted specifically on its design. However, given the focus of the study, this was unlikely to have had a major impact on the overall findings.

The study compared current clinical practice with a NMJ targeting/higher volume technique. As a result, both the targeted injection and the increased volume (resulting in 
wider spread) could contribute to the effect on the spastic muscle in the NMJ group. Both treatment arms experienced a high response rate to treatment, suggesting that a much larger study population is needed to be able to detect any difference, if present at all. Different injection guidance techniques (electric stimulation and EMG) were allowed, to reflect the situation in real-world clinical practice. In an attempt to increase recruitment, a protocol amendment was also made allowing use of US guidance techniques; however, only one patient was injected using this technique. Given that the two main injection guidance techniques well balanced between groups, the specific technique used during the study was likely to have had only a limited impact on the overall results.

In summary, the current study provides further insights in the discussions of the effectiveness of BoNT injections targeted to NMJs. Since this strategy enables patients to be treated using fewer injections, the approach may minimise the potentially painful injection experience while having a similar effect on spasticity. Furthermore, the high number of responders with both injection techniques indeed confirms aboBoNT-A to be an effective treatment option for spasticity. Thus, any measures that could have an effect on implementation and/or compliance are important.

This study was conducted on flexors in the spastic arm, and the effect on other spastic muscles using NMJ-targeted injections has yet to be substantiated. Several previous publications suggest injections near NMJs, but an implementation barrier is the limited number of muscles with known localisation of muscle endplates. ${ }^{16}{ }^{17}{ }^{31-34}$ However, in light of the expanding support for NMJ-targeted injections, further studies adding information to the field are warranted.

\section{Author affiliations}

${ }^{1}$ Department of Neurology, Haukeland University Hospital, Bergen, Norway ${ }^{2}$ Department of Neurology, Spasticity Clinic, Rigshospitalet Glostrup, Glostrup, Denmark

${ }^{3}$ Department of Neurology, University Hospital of Copenhagen, Roskilde Sygehus,

Denmark

${ }^{4}$ Department of Neurology, Vejle Hospital, Vejle, Denmark

${ }^{5}$ Department of Neurology, Aarhus University Hospital, Aarhus, Denmark

${ }^{6}$ Department of Rehabilitation Medicine, Sahlgrenska University Hospital, Gothenburg, Sweden

${ }^{7}$ Medical department, Institut Produits Synthese (AB), Stockholm, Sweden

${ }^{8}$ Ipsen Innovation, Ipsen, Les Ulis, France

${ }^{9}$ Clinic of Dystonia, Bispjeberg Hospital, Copenhagen, Denmark

Acknowledgements The authors thank the investigators and patients participating in this study. The authors thank PCG for assisting in data management. Medical writing support was provided by Nordic Health Economics and funded by Ipsen AB. The Article Processing Charge was paid by Ipsen AB.

Contributors TR and BB-S performed the experiments, analysed the data and wrote the paper. TU and TD conceived and designed the experiments, performed the experiments and analysed the data. RB conceived and designed the experiments, analysed the data and wrote the paper. JH, OJV and PBC performed the experiments. TS conceived and designed the experiments, analysed the data. PM analysed the data. PM conceived and designed the experiments, analysed the data and contributed analysis tools.

Funding The study was sponsored by Ipsen.

Competing interests The authors have, during the last 3 years, received educational support from the sponsor, Ipsen $\mathrm{AB}$ and/or served as consultants for the sponsor. The sponsor contributed to the design of the study; in the collection, analyses/interpretation of data; in the writing of the manuscript and in the decision to publish the results.

Patient consent for publication Not required.

Ethics approval The study received ethical approval from the committee for Scientific Etichs (De videnskapetiske Komiteer for Region Hovedstaden) Kongens Vænge 2, 3400 Hilleröd, Denmark (21 February 2012, 20 June 2012 and 04 July 2014); Central Finland Hospital District, Ethical Commission (KeskiSuomen sairaanhoitopiiri, Eettinen toimikunta), Sairaanhoitopiirin toimisto Rak. 6/2, Keskussairaalantie 19, 40620 Jyväskylä, Finland (27 January 2012 and 27 August 2012); the regional Ethics Committee in Stockholm (Regionala Etikprövningsnämnden i Stockholm), FE289, 17177 Stockholm, Sweden (01 February 2012, 20 June 2012 and 04 July 2014) and the Regional Committee for Medical and Health Ethics south east Norway (Regional komité for medisinsk og, helsefaglig forskningsetikk REK, sør-øst- Norge), P0-box 1130, 0318 0slo, Norway (07 August 2012, 01 November 2012 and 04 July 2014). The study was registered on clinicaltrials.gov on 12 September 2012 (NCT01682148).

Provenance and peer review Not commissioned; externally peer reviewed.

Data sharing statement Trial data analysed during the reported study are available to qualified researchers. Reasonable requests should be directed to the corresponding author.

Open access This is an open access article distributed in accordance with the Creative Commons Attribution Non Commercial (CC BY-NC 4.0) license, which permits others to distribute, remix, adapt, build upon this work non-commercially, and license their derivative works on different terms, provided the original work is properly cited, appropriate credit is given, any changes made indicated, and the use is non-commercial. See: http://creativecommons.org/licenses/by-nc/4.0/.

\section{REFERENCES}

1. Pandyan AD, Gregoric M, Barnes MP, et al. Spasticity: clinical perceptions, neurological realities and meaningful measurement. Disabil Rehabil 2005;27:2-6.

2. Wissel J, Manack A, Brainin M. Toward an epidemiology of poststroke spasticity. Neurology 2013;80:S13-S19.

3. Simpson DM, Hallett M, Ashman EJ, et al. Practice guideline update summary: Botulinum neurotoxin for the treatment of blepharospasm, cervical dystonia, adult spasticity, and headache: Report of the Guideline Development Subcommittee of the American Academy of Neurology. Neurology 2016;86:1818-26.

4. Wissel J, Ward AB, Erztgaard P, et al. European consensus table on the use of botulinum toxin type A in adult spasticity. $J$ Rehabil Med 2009;41:13-25.

5. Bakheit AM, Thilmann AF, Ward AB, et al. A randomized, doubleblind, placebo-controlled, dose-ranging study to compare the efficacy and safety of three doses of botulinum toxin type A (Dysport) with placebo in upper limb spasticity after stroke. Stroke 2000;31:2402-6.

6. Demetrios M, Khan F, Turner-Stokes L, et al. Multidisciplinary rehabilitation following botulinum toxin and other focal intramuscular treatment for post-stroke spasticity. Cochrane Database Syst Rev 2013:CD009689. (published Online First: 07 Jun 2013).

7. Gracies JM, Brashear A, Jech R, et al. Safety and efficacy of abobotulinumtoxinA for hemiparesis in adults with upper limb spasticity after stroke or traumatic brain injury: a double-blind randomised controlled trial. Lancet Neurol 2015;14:992-1001.

8. Rosales RL, Kong KH, Goh KJ, et al. Botulinum toxin injection for hypertonicity of the upper extremity within 12 weeks after stroke: a randomized controlled trial. Neurorehabil Neural Repair 2012;26:812-21.

9. Yelnik AP, Colle FM, Bonan IV, et al. Treatment of shoulder pain in spastic hemiplegia by reducing spasticity of the subscapular muscle: a randomised, double blind, placebo controlled study of botulinum toxin A. J Neurol Neurosurg Psychiatry 2007;78:845-8.

10. Gracies JM, O'Dell M, Vecchio M, et al. Effects of repeated abobotulinumtoxinA injections in upper limb spasticity. Muscle Nerve 2018;57.

11. Ashford S, Turner-Stokes L. Systematic review of upper-limb function measurement methods in botulinum toxin intervention for focal spasticity. Physiother Res Int 2013;18:178-89.

12. Kaku M, Simpson DM. Spotlight on botulinum toxin and its potential in the treatment of stroke-related spasticity. Drug Des Devel Ther 2016;10:1085-99.

13. Lamb YN, Scott LJ. IncobotulinumtoxinA: A Review in Upper Limb Spasticity. Drugs 2016;76:1373-9. 
14. Chan AK, Finlayson $H$, Mills PB. Does the method of botulinum neurotoxin injection for limb spasticity affect outcomes? A systematic review. Clin Rehabil 2017;31:713-21.

15. Grigoriu Al, Dinomais M, Rémy-Néris $O$, et al. Impact of injectionguiding techniques on the effectiveness of botulinum toxin for the treatment of focal spasticity and dystonia: A systematic review. Arch Phys Med Rehabil 2015;96:2067-78.

16. Amirali A, Mu L, Gracies JM, et al. Anatomical localization of motor endplate bands in the human biceps brachii. J Clin Neuromuscul Dis 2007:9:306-12

17. Aquilonius SM, Askmark H, Gillberg PG, et al. Topographical localization of motor endplates in cryosections of whole human muscles. Muscle Nerve 1984;7:287-93.

18. Guzmán-Venegas RA, Araneda OF, Silvestre RA. Differences between motor point and innervation zone locations in the biceps brachii. An exploratory consideration for the treatment of spasticity with botulinum toxin. J Electromyogr Kinesiol 2014;24:923-7.

19. Childers MK, Kornegay JN, Aoki R, et al. Evaluating motor endplate-targeted injections of botulinum toxin type $A$ in a canine model. Muscle Nerve 1998;21:653-5.

20. Gracies JM, Lugassy M, Weisz DJ, et al. Botulinum toxin dilution and endplate targeting in spasticity: a double-blind controlled study. Arch Phys Med Rehabil 2009;90:9-16.

21. Shaari CM, Sanders I. Quantifying how location and dose of botulinum toxin injections affect muscle paralysis. Muscle Nerve 1993;16:964-9.

22. Esquenazi A, Mayer NH. Instrumented assessment of muscle overactivity and spasticity with dynamic polyelectromyographic and motion analysis for treatment planning. Am J Phys Med Rehabil 2004;83:S19-S29.

23. Hefter $\mathrm{H}$, Jost $\mathrm{WH}$, Reissig $\mathrm{A}$, et al. Classification of posture in poststroke upper limb spasticity: a potential decision tool for botulinum toxin A treatment? Int J Rehabil Res 2012;35:227-33.

24. Pacha Vicente D, Forcada Calvet P, Carrera Burgaya A, et al. Innervation of biceps brachii and brachialis: Anatomical and surgical approach. Clin Anat 2005;18:186-94.
25. Saitou K, Masuda T, Michikami D, et al. Innervation zones of the upper and lower limb muscles estimated by using multichannel surface EMG. J Hum Ergol 2000;29:35-52.

26. Kanovský P, Slawek J, Denes Z, et al. Efficacy and safety of botulinum neurotoxin NT 201 in poststroke upper limb spasticity. Clin Neuropharmacol 2009;32:259-65.

27. Hoare BJ, Wallen MA, Imms $\mathrm{C}$, et al. Botulinum toxin $\mathrm{A}$ as an adjunct to treatment in the management of the upper limb in children with spastic cerebral palsy (UPDATE). Cochrane Database Syst Rev 2010:CD003469. (published Online First: 22 Jan 2010).

28. Placzek R, Siebold D, Funk JF. Development of treatment concepts for the use of botulinum toxin a in children with cerebral palsy. Toxins 2010;2:2258-71.

29. Nott MT, Barden HL, Baguley IJ. Goal attainment following upperlimb botulinum toxin-A injections: are we facilitating achievement of client-centred goals? J Rehabil Med 2014;46:864-8.

30. Paracka L, Kollewe K, Dengler R, et al. Botulinum toxin therapy for hyperhidrosis: reduction of injection site pain by nitrous oxide/ oxygen mixtures. J Neural Transm 2015;122:1279-82.

31. Kim MW, Kim JH, Yang YJ, et al. Anatomic localization of motor points in gastrocnemius and soleus muscles. Am J Phys Med Rehabil 2005;84:680-3.

32. Lepage D, Parratte B, Tatu L, et al. Extra- and intramuscular nerve supply of the muscles of the anterior antebrachial compartment: applications for selective neurotomy and for botulinum toxin injection. Surg Radiol Anat 2005;27:420-30.

33. Parratte B, Tatu L, Vuillier F, et al. Intramuscular distribution of nerves in the human triceps surae muscle: anatomical bases for treatment of spastic drop foot with botulinum toxin. Surg Radiol Anat 2002;24:91-6.

34. Santamato A, Micello MF, Panza F, et al. Can botulinum toxin type $A$ injection technique influence the clinical outcome of patients with post-stroke upper limb spasticity? A randomized controlled trial comparing manual needle placement and ultrasound-guided injection techniques. J Neurol Sci 2014;347:39-43. 